\title{
ON RICCATI EQUATIONS IN BANACH ALGEBRAS
}

\author{
RUTH CURTAIN AND AMOL SASANE
}

\begin{abstract}
Let $R$ be a commutative complex Banach algebra with the involution ** and suppose that $A \in R^{n \times n}, B \in R^{n \times m}, C \in R^{p \times n}$. The question of when the Riccati equation

$$
P B B^{\star} P-P A-A^{\star} P-C^{\star} C=0
$$

has a solution $P \in R^{n \times n}$ is investigated. A counterexample to a previous result in the literature on this subject is given, followed by sufficient conditions on the data guaranteeing the existence of such a $P$. Finally, applications to spatially distributed systems are discussed.
\end{abstract}

\section{INTRODUCTION}

If $A \in \mathbb{C}^{n \times n}, B \in \mathbb{C}^{n \times m}$ and $C \in \mathbb{C}^{p \times m}$, then the Riccati equation is

$$
P B B^{*} P-P A-A^{*} P-C^{*} C=0
$$

in the unknown $P \in \mathbb{C}^{n \times n}$. This is a fundamental equation associated with the problem of optimal control of linear control systems with a quadratic cost, and the following is a well known result about the existence of solutions.

Proposition 1.1. Let $A \in \mathbb{C}^{n \times n}, B \in \mathbb{C}^{n \times m}, C \in \mathbb{C}^{p \times n}$ be such that $(A, B)$ is stabilizable and $(A, C)$ is detectable. Then there is a unique positive semidefinite solution $P \in \mathbb{C}^{n \times n}$ to the Riccati equation

$$
P B B^{*} P-P A-A^{*} P-C^{*} C=0,
$$

such that $A-B B P^{*}$ is exponentially stable, that is, $\operatorname{Re}(\lambda)<0$ for all eigenvalues $\lambda$ of $A-B B P^{*}$.

(Recall that the pair $(A, B)$ is stabilizable if there exists a $F \in \mathbb{C}^{m \times n}$ such that $A+B F$ is asymptotically stable, and the pair $(A, C)$ is detectable if the pair $\left(A^{*}, C^{*}\right)$ is stabilizable.)

There has been old (see [3]) and recent (see [4]) renewed interest in the following question: if the data $A, B, C$ have entries in a Banach algebra, then does there exist a solution $P$ also with entries from the same Banach algebra? In this article, we investigate this question. We begin by fixing some notation.

1991 Mathematics Subject Classification. Primary 46J05; Secondary 93D15, 58C15, $47 \mathrm{~N} 20$.

Key words and phrases. Riccati equations, Banach algebras, Systems over rings, Optimal control, Spatially distributed dynamical systems. 
Notation 1.2. Throughout the article, $R$ will denote a commutative, unital, complex, semisimple Banach algebra, which possesses an involution ${ }^{\star}$.

On the other hand, the usual adjoint of a matrix $M=\left[m_{i j}\right] \in \mathbb{C}^{p \times m}$ will be denoted by $M^{*} \in \mathbb{C}^{m \times p}$, that is, $M^{*}=\left[\overline{m_{j i}}\right]$.

$M(R)$ will denote the maximal ideal space of $R$, equipped with the weak-* topology. For $x \in R$, we will denote its Gelfand transform by $\widehat{x}$, that is,

$$
\widehat{x}(\varphi)=\varphi(x), \quad \varphi \in M(R), x \in R .
$$

For a matrix $M \in R^{p \times m}$, whose entry in the $i$ th row and $j$ th column is denoted by $m_{i j}$, we define $M^{\star} \in R^{m \times p}$ to be the matrix whose entry in the $i$ th row and $j$ th column is $m_{j i}^{\star}$. Also by $\widehat{M}$ we mean the $p \times m$ matrix, whose entry in the $i$ th row and $j$ th column is the continuous function $\widehat{m_{i j}}$ on $M(R)$. Summarizing, if $M=\left[m_{i j}\right] \in R^{p \times m}$, then

$$
\begin{aligned}
M^{\star} & =\left[m_{j i}^{\star}\right] \in R^{m \times p}, \\
\widehat{M} & =\left[\widehat{m_{i j}}\right] \in(C(M(R) ; \mathbb{C}))^{p \times m}, \\
(\widehat{M}(\varphi))^{*} & =\left[\widehat{\widehat{m_{j i}}(\varphi)}\right] \in \mathbb{C}^{p \times m} .
\end{aligned}
$$

The following claim was made in [3, Theorem 2.2, p.248].

Claim 1.3. Let $A \in R^{n \times n}, B \in R^{n \times m}, C \in R^{p \times n}$ be such that for all $\varphi \in M(R),(\widehat{A}(\varphi), \widehat{B}(\varphi))$ and $\left(\widehat{A^{\star}}(\varphi), \widehat{C^{\star}}(\varphi)\right)$ are controllable. Then there exists a solution $P \in R^{n \times n}$ such that

$$
P B B^{\star} P-P A-A^{\star} P-C^{\star} C=0 .
$$

(Recall that for matrices $A \in \mathbb{C}^{n \times n}$ and $B \in \mathbb{C}^{n \times m}$, the pair $(A, B)$ is said to be controllable if rank $\left.\left[\begin{array}{lllll}B & A B & A^{2} B & \ldots & A^{n-1} B\end{array}\right]=n.\right)$

However, in Section 2, we will see a counterexample to Claim 1.3, showing that this is not true in general, without invoking extra assumptions, and this is our main result:

Theorem 1.4. Let $A \in R^{n \times n}, B \in R^{n \times m}, C \in R^{p \times n}$ satisfy the following: for all $\varphi \in M(R)$,

(A1) $\widehat{\left(A^{\star}\right)}(\varphi)=(\widehat{A}(\varphi))^{*}$,

(A2) $\widehat{\left(B B^{\star}\right)}(\varphi)=\widehat{B}(\varphi)(\widehat{B}(\varphi))^{*}$,

(A3) $\widehat{\left(C^{\star} C\right)}(\varphi)=(\widehat{C}(\varphi))^{*} \widehat{C}(\varphi)$,

(A4) $(\widehat{A}(\varphi), \widehat{B}(\varphi))$ is stabilizable,

(A5) $(\widehat{A}(\varphi), \widehat{C}(\varphi))$ is detectable.

Then there exists a $P \in R^{n \times n}$ such that

(1) $P B B^{\star} P-P A-A^{\star} P-C^{\star} C=0$,

(2) $A-B B^{\star} P$ is exponentially stable, and

(3) for all $\varphi \in M(R), \widehat{P}(\varphi)$ is positive semidefinite.

In the following we define what is meant by "exponentially stable". 
Definition 1.5. Let $R$ be a commutative, unital, complex, semisimple Banach algebra. If $A \in R^{n \times n}$, let $M_{A}: R^{n} \rightarrow R^{n}$ be the multiplication map by the matrix $A$, that is, $v \mapsto A v\left(v \in R^{n}\right)$. Then $R^{n \times n}$ is a unital complex Banach algebra (for example) with the norm

$$
\|A\|:=\left\|M_{A}\right\|_{\mathcal{L}\left(R^{n}\right)} \quad\left(A \in R^{n \times n}\right)
$$

where $\mathcal{L}\left(R^{n}\right)$ denotes the set of all continuous linear transformations from $R^{n}$ to $R^{n}$, and $R^{n}$ is the Banach space equipped (for example) with the norm

$$
\|x\|=\max \left\{\left\|x_{k}\right\|: 1 \leq k \leq n\right\} \text { for } x=\left[\begin{array}{c}
x_{1} \\
\vdots \\
x_{n}
\end{array}\right],
$$

and $\mathcal{L}\left(R^{n}\right)$ is equipped with the usual operator norm:

$$
\left\|M_{A}\right\|_{\mathcal{L}\left(R^{n}\right)}=\sup \left\{\|A v\|: v \in R^{n} \text { with }\|v\| \leq 1\right\} .
$$

For $A \in R^{n \times n}$, we define

$$
e^{A}=\sum_{k=0}^{\infty} \frac{1}{k !} A^{k} .
$$

The absolute convergence of this series is established just as in the scalar case.

$A \in R^{n \times n}$ is said to be exponentially stable if there exist positive constants $C$ and $\epsilon$ such that

$$
\left\|e^{t A}\right\| \leq C e^{-\epsilon t} \text { for all } t \geq 0 .
$$

Lemma 1.6. Let $A \in R^{n \times n}$. Then the following are equivalent:

(1) $\lambda$ belongs to the spectrum of $A \in R^{n \times n}$.

(2) $\lambda$ belongs to the spectrum of $M_{A} \in \mathcal{L}\left(R^{n}\right)$.

(3) $\lambda$ belongs to the spectrum of $\widehat{A}(\varphi)$ for some $\varphi \in M(R)$.

Proof. The equivalence of (1) and (3) follows from the fact [8, Theorem 8.1, p.830] that $A \in R^{n \times n}$ is invertible in the Banach algebra $R^{n \times n}$ if and only if the matrix $\widehat{R}(\varphi)$ is invertible in $\mathbb{C}^{n \times n}$ for each $\varphi \in M(R)$. For $A \in R^{n \times n}$, it can be seen that $A$ is invertible in $R^{n \times n}$ if and only if $M_{A}$ is invertible in $\mathcal{L}\left(R^{n}\right)$. Indeed, the 'only if' part is trivial, since if $A A^{-1}=I=A^{-1} B$, then $M_{A} M_{A^{-1}}=I=M_{A^{-1}} M_{A}$. Vice versa, if $M_{A} T=I=T M_{A}$ for some $T \in \mathcal{L}\left(R^{n}\right)$, then set

$$
A_{:}^{-1}=\left[\begin{array}{lll}
T e_{1} & \ldots & T e_{n}
\end{array}\right] \in R^{n \times n},
$$

where $e_{k}(k=1, \ldots, n)$ denotes the vector in $R^{n}$ with 1 in the $k$ th position and zeros elsewhere. Then $M_{A^{-1}} e_{k}=T e_{k}$ for all $k=1, \ldots, n$, and so $M_{A^{-1}} v=T v$ for all $v \in R^{n}$. Hence (1) and (2) are equivalent.

The following gives a characterization of exponential stability. 
Proposition 1.7. Let $A \in R^{n \times n}$. Then $A$ is exponentially stable if and only if

$\sup \{\operatorname{Re}(\lambda): \lambda$ is an eigenvalue of $\widehat{A}(\varphi)$ for some $\varphi \in M(R)\}<0$.

Proof. We recall the result in semigroup theory that the semigroup generated by a continuous linear transformation on a Banach space is exponentially stable if and only if the supremum of the real parts of points in the spectrum of the operator is strictly negative [6, Corollary IV.2.4, p.252 and Proposition V.1.7, p.299]. Using this, we see that $A$ is exponentially stable if and only if $\sup \left\{\operatorname{Re}(\lambda): \lambda\right.$ belongs to the spectrum of $\left.M_{A} \in \mathcal{L}\left(R^{n}\right)\right\}$ is negative. The proof is now finished by using Lemma 1.6 .

The proof in Section 3 of our main result above is similar to the approach in [3], where we first take Gelfand transform of our equation, and show that the pointwise solution is continuous. Then we use the Banach algebra operational calculus to ensure that this continuous solution is actually the Gelfand transform of a matrix with entries from the Banach algebra.

In Section 4 we discuss the applications of this result to the control of spatially invariant systems.

\section{Counterexample to Claim 1.3}

Example 2.1. Consider the Banach algebra $C^{1}(\mathbb{T})$ of all continuously differentiable functions on the unit circle with pointwise operations and the norm

$$
\|f\|_{C^{1}(\mathbb{T})}=\|f\|_{\infty}+\left\|f^{\prime}\right\|_{\infty},
$$

with the understanding that $f^{\prime}\left(e^{i \theta}\right):=\frac{d F}{d \theta}(\theta)$, where $F(\theta):=f\left(e^{i \theta}\right)(\theta \in \mathbb{R})$. Then $C^{1}(\mathbb{T})$ is a semisimple commutative unital complex Banach algebra. Every point $z$ on $\mathbb{T}$ gives rise to the complex homomorphism

$$
f \mapsto f(z): C^{1}(\mathbb{T}) \rightarrow \mathbb{C} .
$$

Also, all complex homomorphisms arise in this manner, and this can be seen as follows. If $\varphi$ is a complex homomorphism which is not a point evaluation at any point of $\mathbb{T}$, then given any $z \in \mathbb{T}$, there is a corresponding $f \in C^{1}(\mathbb{T})$ such that $\varphi(f)=0$, but $f(z) \neq 0$. So in fact for $w$ 's belonging to a small neighbourhood of this $z$, we have $|f(w)| \geq \delta_{z}>0$. But $\mathbb{T}$ is compact, and so there exist finitely many functions $f_{1}, \ldots, f_{n}$ such that $\varphi\left(f_{i}\right)=0$ for each $i$, and for every point on the unit circle, at least one of the functions $f_{i}$ $(1 \leq i \leq n)$ is nonzero there. Thus the function

$$
g:=f_{1} \overline{f_{1}}+\cdots+f_{n} \overline{f_{n}}
$$

is in $C^{1}(\mathbb{T})$, it satisfies $\varphi(f)=0$, and is nonzero on $\mathbb{T}$. But being nonzero on $\mathbb{T}, g$ is invertible as an element of $C^{1}(\mathbb{T})$, a contradiction to the fact that a maximal ideal cannot contain units. Hence the maximal ideal space of $C^{1}(\mathbb{T})$ can be identified with the unit circle $\mathbb{T}$. 
The Banach algebra $C^{1}(\mathbb{T})$ possesses the involution * ${ }^{\star}$ defined by

$$
f^{\star}(z)=\overline{f(\bar{z})}(z \in \mathbb{T}), \text { for } f \in C^{1}(\mathbb{T}) .
$$

Now consider the following $A, B, C \in C^{1}(\mathbb{T})$ :

$$
A(z)=z, \quad B(z)=1, \quad C(z)=1, \quad(z \in \mathbb{T}) .
$$

Then we have that $A=A^{\star}, B=B^{\star}=C=C^{\star}$.

For each $z \in \mathbb{T},(\widehat{A}(\varphi), \widehat{B}(\varphi))=(z, 1)=\left(\widehat{A^{\star}}(\varphi), \widehat{C^{\star}}(\varphi)\right)$ is controllable. Thus all the hypotheses of the Claim 1.3 are satisfied. But we will show below that the corresponding Riccati equation has no solution in the Banach algebra $C^{1}(\mathbb{T})$.

The Riccati equation is $P^{2}-2 z P-1=0$. Let us suppose that this has a solution $P \in C^{1}(\mathbb{T})$. Then we obtain

$$
(\widehat{P}(z))^{2}-2 z \widehat{P}(z)-1=0 \quad(z \in \mathbb{T}),
$$

that is $(\widehat{P}(z)-z)^{2}=z^{2}+1(z \in \mathbb{T})$. We will now show the following:

Claim: There is no $Q \in C^{1}(\mathbb{T})$ such that $(\widehat{Q}(z))^{2}=z^{2}+1(z \in \mathbb{T})$.

It is not hard to see that the function $g$ given by

$$
g\left(e^{i \theta}\right):= \begin{cases}\sqrt{2} \sqrt{\cos \theta} e^{i \frac{\theta}{2}} & \text { if } \cos \theta \geq 0 \\ \sqrt{2} \sqrt{-\cos \theta} e^{i \frac{(\theta+\pi)}{2}} & \text { if } \cos \theta<0\end{cases}
$$

does satisfy $(g(z))^{2}=z^{2}+1(z \in \mathbb{T})$. As the function $g$ is not differentiable when $\theta=\frac{\pi}{2}$, it follows that $g \notin C^{1}(\mathbb{T})$. Since $g$ has two roots on $\mathbb{T}$, namely at $i$ and at $-i$, it follows from $Q^{2}=g^{2}$, that $Q$ is either $g$ or $-g$ or $h g$ or $-h g$, where

$$
h(z):= \begin{cases}1 & \text { if } \operatorname{Re}(z) \geq 0 \\ -1 & \text { if } \operatorname{Re}(z)<0 .\end{cases}
$$

But none of these functions is differentiable when $\theta=\frac{\pi}{2}$. This completes the proof of the fact that there is no $Q \in C^{1}(\mathbb{T})$ such that $(\widehat{Q}(z))^{2}=z^{2}+1$ $(z \in \mathbb{T})$.

So we conclude that the Claim 1.3 is false.

\section{Proof of the MAIN RESUlt}

We will need the following two results. The first one says that if we consider the classical Riccati equation with constant complex matricial data $A, B, C$, then the solution $P$ depends continuously on the $A, B, C$; see $[12$, Theorem 1.2.1, p.260].

Proposition 3.1. With the same notation as in Proposition 1.1, the maximal Hermitian solution $P(A, B, C)$ of the Riccati equation is a continuous function of $(A, B, C)$. (Here $P(A, B, C)$ is viewed as a function on a subset of $\mathbb{C}^{n^{2}} \times \mathbb{C}^{n m} \times \mathbb{C}^{\text {pn }}$ with the usual topology $)$.

The next result we will need is the following (see [10, p.155]), and this will be used to pass from continuous functions on $M(R)$ to elements of $R$. 
Proposition 3.2. Let $h_{1}, \ldots, h_{s}$ be continuous functions on $M(R)$. Suppose that $f_{1}, \ldots f_{\ell}$ in $R$ and $G_{1}\left(z_{1}, \ldots, z_{s+\ell}\right), \ldots, G_{t}\left(z_{1}, \ldots, z_{s+\ell}\right)$ are holomorphic functions with $t \geq s$ defined on a neighbourhood of the joint spectrum

$\sigma\left(h_{1}, \ldots, h_{s}, f_{1}, \ldots, f_{\ell}\right):=\left\{\left(h_{1}(\varphi), \ldots, h_{s}(\varphi), \widehat{f}_{1}(\varphi), \ldots, \widehat{f}_{\ell}(\varphi)\right): \varphi \in M(R)\right\}$, such that

$$
G_{k}\left(h_{1}, \ldots, h_{s}, \widehat{f}_{1}, \ldots, \widehat{f}_{\ell}\right)=0 \text { on } M(R) \text { for } 1 \leq k \leq t
$$

If the rank of the Jacobi matrix $\frac{\partial\left(G_{1}, \ldots, G_{t}\right)}{\partial\left(z_{1}, \ldots, z_{s}\right)}$ is s on $\sigma\left(h_{1}, \ldots, h_{s}, f_{1}, \ldots, f_{\ell}\right)$, then there exist elements $g_{1}, \ldots, g_{s}$ in $R$ such that

$$
\widehat{g_{1}}=h_{1}, \ldots, \widehat{g_{s}}=h_{s} .
$$

We are now ready to prove our main result.

Proof of Theorem 1.4. If we fix a $\varphi \in M(R)$, then owing to the assumptions (A4) and (A5), we know that there is a unique solution in $\mathbb{C}^{n \times n}$, which we will denote by $\Pi(\varphi)$, such that it is positive semidefinite,

$$
\Pi(\varphi) \widehat{B}(\varphi)(\widehat{B}(\varphi))^{*} \Pi(\varphi)-\Pi(\varphi) \widehat{A}(\varphi)-(\widehat{A}(\varphi))^{*} \Pi(\varphi)-(\widehat{C}(\varphi))^{*} \widehat{C}(\varphi)=0
$$

and $\widehat{A}(\varphi)-\widehat{B}(\varphi)(\widehat{B}(\varphi))^{*} \Pi(\varphi)$ is exponentially stable.

Moreover, from Proposition 3.1, it follows that the map $\varphi \mapsto \Pi(\varphi)$ is continuous on $M(R)$.

Finally we will apply Proposition 3.2. We have in our case $s=n^{2}$, $t=n^{2}$, the $h_{i}$ 's are the components of $\Pi$ and the $f_{i}$ 's are the components of $A, A^{\star}, B B^{\star}, C^{\star} C$ (which are totally $\ell=n^{2}+n^{2}+n^{2}+n^{2}=4 n^{2}$ in number). The maps $G_{1}, \ldots G_{t=n^{2}}$ are the $n^{2}$ components of the map

$$
(\Theta, U, V, W, X) \mapsto \Theta W \Theta-\Theta U-V \Theta-X .
$$

(In the above we have the replacements of $A, A^{*}, B B^{*}, C C^{*}$ by the complex variables which are the components of $U, V, W, X$, respectively. The replacements of the $P$ in the Riccati equation is by the complex variables which are the components of $\Theta$.) Clearly the above map is holomorphic not just on the joint spectrum, but rather in the whole of $\mathbb{C}^{s+\ell}=\mathbb{C}^{n^{2}+4 n^{2}}$.

In light of the assumptions (A1)-(A3) and and (3.2), we know that there is a continuous solution $\Pi$ on the maximal ideal space such that for all $k$,

$$
G_{k}\left(\Pi, \widehat{A}, \widehat{A^{\star}}, \widehat{B B^{\star}}, \widehat{C^{\star} C}\right)=0
$$

on $M(R)$ (that is, condition (3.1) in Proposition 3.2 is satisfied).

So we now investigate the Jacobian with respect to the variables in $\Theta$. The Jacobian with respect to the $\Theta$ variables at the point

$\left(\Pi(\varphi), \widehat{A}(\varphi), \quad(\widehat{A}(\varphi))^{*}, \widehat{B B^{\star}}(\varphi)=\widehat{B}(\varphi)(\widehat{B}(\varphi))^{*}, \widehat{C^{\star} C}(\varphi)=(\widehat{C}(\varphi))^{*} \widehat{C}(\varphi)\right)$

is the following linear transformation $\Lambda$ from $\mathbb{C}^{n^{2}} \rightarrow \mathbb{C}^{n^{2}}$ :

$$
\Theta \mapsto \Theta \widehat{B}(\varphi)(\widehat{B}(\varphi))^{*} \Pi(\varphi)+\Pi(\varphi) \widehat{B}(\varphi)(\widehat{B}(\varphi))^{*} \Theta-\Theta \widehat{A}(\varphi)-(\widehat{A}(\varphi))^{*} \Theta,
$$


that is,

$$
\Theta \mapsto-\left(\left(\widehat{A}(\varphi)-\widehat{B}(\varphi)(\widehat{B}(\varphi))^{*} \Pi(\varphi)\right)^{*} \Theta+\Theta\left(\widehat{A}(\varphi)-\widehat{B}(\varphi)(\widehat{B}(\varphi))^{*} \Pi(\varphi)\right)\right)
$$

The set of eigenvalues of $\Lambda$ consists of the numbers

$$
-(\bar{\lambda}+\mu)
$$

where $\lambda, \mu$ belong to the set of eigenvalues of $\widehat{A}(\varphi)-\widehat{B}(\varphi)(\widehat{B}(\varphi))^{*} \Pi(\varphi)$; see for example [2, Proposition 7.2.3]. But since $\widehat{A}(\varphi)-\widehat{B}(\varphi)(\widehat{B}(\varphi))^{*} \Pi(\varphi)$ is exponentially stable, all its eigenvalues have a negative real part. Hence $-(\bar{\lambda}+\mu) \neq 0$ for all $\lambda, \mu$ belonging to the set of eigenvalues of the matrix $\widehat{A}(\varphi)-\widehat{B}(\varphi)(\widehat{B}(\varphi))^{*} \Pi(\varphi)$. Consequently, the map $\Lambda$ is invertible from $\mathbb{C}^{n^{2}}$ to $\mathbb{C}^{n^{2}}$, and its rank is $n^{2}=s$. So by Proposition 3.2. there exists a $P \in R^{n \times n}$ such that $\widehat{P}(\varphi)=\Pi(\varphi)$ for all $\varphi \in M(R)$. From (3.3), it follows (using the fact that $R$ is semisimple) that

$$
P B B^{\star} P-P A-A^{\star} P-C^{\star} C=0 .
$$

From the property possessed by the pointwise solutions $\Pi(\varphi)(\varphi \in M(R))$ of the constant complex matricial Riccati equations (3.2), we have that for all $\varphi \in M(R)$, all eigenvalues of $\left(A \widehat{-B B^{\star}} P\right)(\varphi)$ have a negative real part. But the set-valued map taking a square complex matrix of size $n \times n$ to its spectrum (a set of $n$ complex numbers) is a continuous map; see for example [11, II, $\S 5$, Theorem 5.14, p.118]. Since $M(R)$ is compact in the Gelfand topology (the weak-* topology induced on $M(R)$ considered as a subset of $\mathcal{L}(R ; \mathbb{C}))$, it follows that

$\sup \left\{\operatorname{Re}(\lambda): \lambda\right.$ is an eigenvalue of $\left(\widehat{A-B B^{\star}} P\right)(\varphi)$ for some $\left.\varphi \in M(R)\right\}<0$. From Proposition [1.7, it follows that $A-B B^{\star} P$ is exponentially stable. Finally, again by the property possessed by the pointwise solution $\Pi$, we have that for all $\varphi \in M(R), \widehat{P}(\varphi)$ is positive semidefinite. This completes the proof of Theorem 1.4 .

Example 3.3 (Example 2.1 revisited). Let us check what went wrong with our example considered earlier. Recall that the Banach algebra was $C^{1}(\mathbb{T})$, the involution $*^{\star}$ was given by

$$
f^{\star}(z)=\overline{f(\bar{z})} \quad(z \in \mathbb{T})
$$

and the Riccati equation data was given by $A=z, B=C=1$. We see that in Theorem 1.4, for this particular example, although the assumptions (A2)-(A5) are satisfied, (A1) fails to hold. Indeed,

$$
A^{\star}(z)=z \quad(z \in \mathbb{T})
$$

and so for $z \in \mathbb{T} \backslash\{-1,1\}$, we have

$$
\widehat{\left(A^{\star}\right)}(z)=z \neq \bar{z}=(\widehat{A}(z))^{*} .
$$

So it is no surprise that a solution does not exist to the Riccati equation. 
If instead, we consider the following new $A$, given by

$$
A(z)=z+\frac{1}{z} \quad(z \in \mathbb{T})
$$

then

$$
\widehat{\left(A^{\star}\right)}(z)=z+\frac{1}{z}=\overline{z+\frac{1}{z}}=(\widehat{A}(z))^{*} \quad(z \in \mathbb{T}) .
$$

With the same $B=C=1$ considered earlier, we see that the assumptions (A1)-(A5) in Theorem 1.4 are all satisfied now, and the Riccati equation

$$
P B B^{\star} P-P A-A^{\star} P-C^{\star} C=P^{2}-2\left(z+\frac{1}{z}\right) P-1=0
$$

has a solution $P \in C^{1}(\mathbb{T})$, given by:

$$
P\left(e^{i \theta}\right)=2 \cos \theta+\sqrt{(2 \cos \theta)^{2}+1} \quad(\theta \in \mathbb{R}) .
$$

Clearly, $P\left(e^{i \theta}\right)$ is positive semidefinite (as it is $\geq 0$ ). Moreover,

$$
\left(A \widehat{-B B^{\star}} P\right)\left(e^{i \theta}\right)=-\sqrt{(2 \cos \theta)^{2}+1} \leq-1,
$$

and so $A-B B^{\star} P$ is exponentially stable by Proposition 1.7 .

We observe that whether or not assumptions (A1)-(A3) in Theorem 1.4 hold is intimately related to the choice of the involution .* in the Banach algebra $R$. For some commutative Banach algebras with involutions, this is automatic, namely if it is symmetric.

Definition 3.4. A unital Banach algebra $R$ with an involution $\cdot^{\star}$ is said to be symmetric if for every $x \in R$, the spectrum of $x x^{\star}$ (as an element of $R$ ) is contained in $[0,+\infty)$. Equivalently, $R$ is symmetric if and only if for every $x \in R$ satisfying $x=x^{\star}$ implies that the spectrum of $x$ is real; see [9, $\S 2.3$, p.2700]. The involution is then called a symmetric involution.

In the case when $R$ is commutative, this is equivalent to the following (see [7. Definition 2, §I.8, p.57]).

Proposition 3.5. Let $R$ be a commutative unital complex semisimple $B a$ nach algebra with an involution $\cdot{ }^{\star}$. Then the following are equivalent:

(1) $R$ is symmetric.

(2) For each $x \in R, \varphi\left(x^{\star}\right)=\overline{\varphi(x)}(\varphi \in M(R))$.

(3) For each $x \in R, 1+x^{\star} x$ is invertible in $R$.

Proof. The equivalence of (2) and (3) is precisely [7, Theorem 2, p.59].

Let us now show that (1) and (2) are equivalent. Suppose that (1) holds. Let $x \in R$. Then using the fact that the spectrum of an element is the range of its Gelfand transform, it follows that

$$
\left(\widehat{1+x^{\star}} x\right)(\varphi)=1+\widehat{x^{\star} x}(\varphi) \geq 1,
$$

and in particular, $\left(\widehat{1+x^{\star}} x\right)(\varphi) \neq 0$ for all $\varphi \in M(R)$. Thus $1+x^{\star} x$ is invertible as an element of $R$. So (3), and consequently also (2), holds. 
Now suppose that (2) holds. Let $x \in R$. We have that

$$
\widehat{x^{\star} x}(\varphi)=\varphi\left(x^{\star} x\right)=\varphi\left(x^{\star}\right) \varphi(x)=\overline{\varphi(x)} \varphi(x)=|\varphi(x)|^{2} \geq 0 .
$$

Since the spectrum of $x^{\star} x$ is the range of its Gelfand transform, it follows that the spectrum of $x^{\star} x$ is contained in the half line $[0,+\infty)$. Thus $R$ is symmetric.

In particular, all $C^{*}$-algebras are symmetric.

We have the following consequence of our main result.

Corollary 3.6. Let $R$ be a commutative unital complex semisimple symmetric Banach algebra with a symmetric involution $\cdot{ }^{\star}$. Let $A \in R^{n \times n}$, $B \in R^{n \times m}, C \in R^{p \times n}$ satisfy the following: for all $\varphi \in M(R)$,

(i) $(\widehat{A}(\varphi), \widehat{B}(\varphi))$ is stabilizable,

(ii) $(\widehat{A}(\varphi), \widehat{C}(\varphi))$ is detectable.

Then there exists a $P \in R^{n \times n}$ such that

(1) $P B B^{\star} P-P A-A^{\star} P-C^{\star} C=0$,

(2) $A-B B^{\star} P$ is exponentially stable, and

(3) $P=P^{\star}$ and the spectrum of $P$ (as an element of the Banach algebra $\left.R^{n \times n}\right)$ is contained in $[0,+\infty)$.

Proof. This is an immediate consequence of Theorem 1.4 since (A1)-(A3) are satisfied automatically owing to the symmetry of the Banach algebra $R$. Also (3) above follows from the conclusion (3) of Theorem 1.4 and the symmetry property of $R$. Indeed we have that for all $\varphi \in M(R)$,

$$
\widehat{P^{\star}}(\varphi)=(\widehat{P}(\varphi))^{*}=\widehat{P}(\varphi),
$$

where the first equality follows from the symmetry of $R$ and the second equality follows from Theorem 1.4.(3). Thus $P^{\star}=P$. The spectrum of $P$ coincides with the set containing the eigenvalues of $\widehat{P}(\varphi)(\varphi \in M(R))$ and since for each $\varphi \in M(R), \widehat{P}(\varphi)$ is positive semidefinite, it follows that the spectrum of $P$ is contained in $[0,+\infty)$.

Example 3.7 (Example 2.1 revisited). Consider the same Banach algebra $C^{1}(\mathbb{T})$ as in Example 2.1, and the same Riccati equation data $A, B, C$ given there, namely $A=z, B=C=1$, but now with a new involution on $C^{1}(\mathbb{T})$, given simply by

$$
f^{\star}(z)=\overline{f(z)} \quad(z \in \mathbb{T}) .
$$

Now the $A$ does satisfy assumption (A1) from Theorem 1.4, since

$$
\widehat{\left(A^{\star}\right)}(z)=\bar{z}=(\widehat{A}(z))^{*} \quad(z \in \mathbb{T}) .
$$

Also, as before the assumptions (A2)-(A5) are satisfied. The corresponding Riccati equation is

$$
P B B^{\star} P-P A-A^{*} P-C^{\star} C=P^{2}-(z+\bar{z}) P-1=0,
$$


and it has a solution $P \in C^{1}(\mathbb{T})$, given by:

$$
P\left(e^{i \theta}\right)=\cos \theta+\sqrt{(\cos \theta)^{2}+1} \quad(\theta \in \mathbb{R}) .
$$

Clearly, $P\left(e^{i \theta}\right)$ is positive semidefinite (as it is $\geq 0$ ). Moreover, from

$$
\left(A \widehat{-B B^{\star}} P\right)(\varphi)=-\sqrt{(\cos \theta)^{2}+1} \leq-1,
$$

it follows that that $A-B B^{\star} P$ is exponentially stable.

\section{Application to Spatially inVARiant systems}

In this section we discuss the applications of our results to control problems for spatially invariant systems introduced in [1]. The analysis of spatially invariant systems can be greatly simplified by taking Fourier transforms, see [1], [5]. This yields systems described by multiplication operators with symbols $A, B, C \in\left(L^{\infty}(\mathbb{T})\right)^{n \times n}$. The Linear Quadratic Regulator (LQR) control design is to use the feedback $F=-B B^{*} P$, where $P$ is the bounded, self-adjoint, stabilizing solution to the LQR Riccati equation (1.1) on the Hilbert space $\left(L^{2}(\mathbb{T})\right)^{n}$. For the design of implementable controllers it is important that the gain operator have a spatially decaying property (see [1]). This translates into the mathematical question of when the LQR Riccati equation (1.1) has a stabilizing solution in a suitable subalgebra (for example, $\left(L^{1}(\mathbb{T})\right)^{n \times n}$ is a subalgebra of $\left.\mathcal{L}\left(\left(L^{2}(\mathbb{T})\right)^{n}\right)=\left(L^{\infty}(\mathbb{T})\right)^{n \times n}\right)$. So the spatially decaying property now translates into finding suitable subalgebras of $\left(L^{\infty}(\mathbb{T})\right)^{n \times n}$, in particular, the weighted Wiener algebras. From our results in the previous sections it suffices to identify the symmetric Wiener algebras for the case $n=1$. In the following example we show that a large class of Wiener subalgebras of $L^{\infty}(\mathbb{T})$ do have this property.

Example 4.1 (Even-weighted Wiener algebras). Let $\alpha=\left(\alpha_{k}\right)_{k \in \mathbb{Z}}$ be any sequence of even weights, that is, the $\alpha_{k}$ 's are positive real numbers satisfying

$$
\alpha_{-k}=\alpha_{k} \quad(k \in \mathbb{Z}) .
$$

Suppose, moreover that

$$
\alpha_{k+l} \leq \alpha_{k} \alpha_{l} \quad(k, l \in \mathbb{Z}) .
$$

Consider the even-weighted Wiener algebra $W_{\alpha}(\mathbb{T})$ of the unit circle $\mathbb{T}$ given by

$$
W_{\alpha}(\mathbb{T})=\left\{f: f(z)=\sum_{k \in \mathbb{Z}} f_{k} z^{k}(z \in \mathbb{T}) \text { and } \sum_{k \in \mathbb{Z}} \alpha_{k}\left|f_{k}\right|<+\infty\right\},
$$

with pointwise operations, and the norm

$$
\|f\|_{W_{\alpha}(\mathbb{T})}=\sum_{k \in \mathbb{Z}} \alpha_{k}\left|f_{k}\right|, \quad f(z)=\sum_{k \in \mathbb{Z}} f_{k} z^{k}(z \in \mathbb{T}) .
$$

Then this is a Banach algebra; see [7, §19.4, p.118-120]. The maximal ideal space of such even-weighted Wiener algebras can be identified with the annulus

$$
\mathbb{A}(\rho)=\{z \in \mathbb{C}: 1 / \rho \leq|z| \leq \rho\},
$$


where $\rho:=\inf _{k>0} \sqrt[k]{\alpha_{k}}=\lim _{k \rightarrow \infty} \sqrt[k]{\alpha_{k}}$. The Gelfand transform is given by

$$
\widehat{f}(z)=\sum_{k \in \mathbb{Z}} f_{k} z^{k} \quad(z \in \mathbb{A}(\rho)) .
$$

When $\rho=1$ the weights given by $\alpha$ are said to satisfy the Gelfand-RaikovShilov condition and the annulus $\mathbb{A}(\rho)$ degenerates to the circle $\mathbb{T}$. Examples that occur in the applications are subexponential weights

$$
\alpha_{k}=e^{\alpha|k|^{\beta}}, \quad \alpha>0, \quad 0 \leq \beta<1,
$$

and polynomial weights

$$
\alpha_{k}=(1+|k|)^{s}, \quad s \geq 0 .
$$

Consider the following involution:

$$
f^{\star}(z)=\overline{f\left(\frac{1}{\bar{z}}\right)} \quad\left(z \in \mathbb{A}(\rho), f \in W_{\alpha}(\mathbb{T})\right) .
$$

Note that under the Gelfand-Raikov-Shilov condition the involution $\cdot^{\star}$ reduces to the following:

$$
f^{\star}(z)=\overline{f(z)} \quad\left(z \in \mathbb{T}, f \in W_{\alpha}(\mathbb{T})\right),
$$

and with this involution $W_{\alpha}(\mathbb{T})$ is a symmetric Banach algebra. Then for matrices $A, B, C$ with entries from $W_{\alpha}(\mathbb{T})$ the assumptions (A1)-(A3) of Theorem 1.4 are automatically satisfied.

We remark that if the Gelfand-Raikov-Shilov condition is not satisfied, then $W_{\alpha}(\mathbb{T})$ is not a symmetric algebra with the involution considered in the previous example.

In the case of spatially invariant systems when the spatial argument is continuous (rather than discrete), the state space is $\left(L^{2}(\mathbb{R})\right)^{n}$ (as opposed to $\left(L^{2}(\mathbb{T})\right)^{n}$ or $\left(\ell^{2}(\mathbb{Z})\right)^{n}$ in the discrete case of the previous example), and the spatially decaying property reduces to asking that the Riccati equation solution belong to appropriate subalgebras of $\left(L^{\infty}(\mathbb{R})\right)^{n \times n}$. In this context, the following example is relevant.

Example 4.2 (Even-weighted Wiener algebra of the line). Let the weight $w: \mathbb{R} \rightarrow(0,+\infty)$ be a continuous function satisfying

$$
w(t+\tau) \leq w(t) w(\tau) \text { and } w(-t)=w(t) \quad(t, \tau \in \mathbb{R}) .
$$

Let $L^{1}(\mathbb{R}, w)$ be the set of all Lebesgue measurable complex valued functions on $\mathbb{R}$ such that

$$
\|f\|_{L^{1}(\mathbb{R}, w)}=\int_{\mathbb{R}}|f(t)| w(t) d t<+\infty .
$$

Then $L(\mathbb{R}, w)$ is a Banach algebra with this norm, with pointwise addition and scalar multiplication, and with the multiplication operation taken to be convolution:

$$
(f * g)(t)=\int_{\mathbb{R}} f(t-\tau) g(\tau) d \tau \quad(t \in \mathbb{R}) .
$$


We adjoin a unit to the Banach algebra $L^{1}(\mathbb{R}, w)$ to obtain its unitization, denoted by $L^{1}(\mathbb{R}, w)+\mathbb{C}$ (see for instance [14, p.246]), and the norm of an element $(f, \zeta) \in L^{1}(\mathbb{R}, w)+\mathbb{C}$ is given by

$$
\|(f, \zeta)\|=\|f\|_{L^{1}(\mathbb{R}, w)}+|\zeta| .
$$

It can be shown that (see [8, (vii), p.816])

$$
\tau:=\inf _{t<0} \frac{\log w(t)}{-t}=\lim _{t \rightarrow-\infty} \frac{\log w(t)}{-t}(\geq 0)
$$

and that every complex homomorphism $\varphi$ on $L^{1}(\mathbb{R}, w)+\mathbb{C}$ is either of one of the following types:

$\underline{1}^{\circ} L^{1}(\mathbb{R}, w) \subset \operatorname{ker} \varphi$. In this case,

$$
\varphi((f, \zeta))=\varphi_{\infty}((f, \zeta)):=\zeta \quad\left(f \in L^{1}(\mathbb{R}, w), \zeta \in \mathbb{C}\right) .
$$

$\underline{2}^{\circ}$ It is not the case that $L^{1}(\mathbb{R}, w) \subset \operatorname{ker} \varphi$. In this case, $\varphi$ is a nontrivial multiplicative linear functional on $L^{1}(\mathbb{R} ; w)$, and so (see [13, p.74]), there exists a complex number $\lambda$ such that $-\tau \leq \operatorname{Im}(\lambda) \leq \tau$ and

$$
\varphi((f, 0))=\int_{\mathbb{R}} f(t) e^{i \lambda t} d t \quad\left(f \in L^{1}(\mathbb{R}, w)\right) .
$$

Consequently,

$$
\varphi((f, \zeta))=\varphi_{\lambda}((f, \zeta)):=\int_{\mathbb{R}} f(t) e^{i \lambda t} d t+\zeta \quad\left(f \in L^{1}(\mathbb{R}, w), \zeta \in \mathbb{C}\right) .
$$

The Gelfand transform of $(f, \zeta)$ is given by

$$
\widehat{(f, \zeta)}(\varphi)= \begin{cases}\int_{\mathbb{R}} f(t) e^{i \lambda t} d t+\zeta & \text { if } \varphi=\varphi_{\lambda},-\tau \leq \operatorname{Im}(\lambda) \leq \tau \\ \zeta & \text { if } \varphi=\varphi_{\infty} .\end{cases}
$$

Consider the involution given by

$$
(f, \zeta)^{\star}=(\overline{f(-\cdot)}, \bar{\zeta}) .
$$

The weight $w$ is said to satisfy the Gelfand-Raikov-Shilov condition if $\tau=0$. In this case, it is easy to check that

$$
\widehat{(f, \zeta)^{\star}}(\varphi)=\widehat{(f, \zeta)(\varphi)}
$$

for all $\varphi \in M\left(L^{1}(\mathbb{R}, w)+\mathbb{C}\right), f \in L^{1}(\mathbb{R}, w)$ and $\zeta \in \mathbb{C}$. Thus $L^{1}(\mathbb{R}, w)+\mathbb{C}$ is a symmetric Banach algebra with this involution. Hence for matrices $A, B, C$ with entries from $L^{1}(\mathbb{R}, w)+\mathbb{C}$, the assumptions (A1)-(A3) of Theorem 1.4 are then automatically satisfied. 


\section{REFERENCES}

[1] B. Bamieh, F. Paganini and M.A. Dahleh. Distributed control of spatially invariant systems. IEEE Trans. Automatic Control 47:1091-1107, 2002.

[2] D.S. Bernstein. Matrix mathematics. Theory, facts, and formulas with application to linear systems theory. Princeton University Press, Princeton, NJ, 2005.

[3] C. Byrnes. Realization theory and quadratic optimal controllers for systems defined over Banach and Frechét algebras. In Proceedings of the 19th IEEE Conference on Decision and Control including the Symposium on Adaptive Processes, December 1980, Volume 19: 247-251.

[4] R.F. Curtain. Algebraic properties of Riccati equations. In Proceedings of the 19th International Symposium on Mathematical Theory of Networks and Systems (MTNS 2010), Budapest, Hungary, 5-9 July, 2010.

[5] R.F. Curtain, O.V. Iftime and H.J. Zwart. System theoretic properties of a class of spatially distributed systems, Automatica 45(7), 1619-1627, 2009.

[6] K-J. Engel and R. Nagel. One-parameter semigroups for linear evolution equations. Graduate Texts in Mathematics, 194. Springer-Verlag, New York, 2000.

[7] I. Gelfand, D. Raikov and G. Shilov. Commutative normed rings. Translated from the Russian, with a supplementary chapter. Chelsea Publishing Co., New York, 1964

[8] I. Gohberg, S. Goldberg and M.A. Kaashoek. Classes of linear operators. Vol. II. Operator Theory: Advances and Applications, 63. Birkhäuser Verlag, Basel, 1993.

[9] K. Gröchenig and M. Leinert. Symmetry and inverse-closedness of matrix algebras and functional calculus for infinite matrices. Transactions of the American Mathematical Society, 358: 2695-2711, 2006.

[10] M. Hayashi. Implicit function theorem for Banach algebras. Journal of the London Mathematical Society. Second Series., (2)13:155-161, no. 1, 1976.

[11] T. Kato. Perturbation theory for linear operators. Reprint of the 1980 edition. Classics in Mathematics. Springer-Verlag, Berlin, 1995.

[12] P. Lancaster and L. Rodman. Algebraic Riccati equations. The Clarendon Press, Oxford University Press, New York, 1995.

[13] L.H. Loomis. An introduction to abstract harmonic analysis. D. Van Nostrand Company, Toronto-New York-London, 1953.

[14] W. Rudin. Functional analysis. Second edition. International Series in Pure and Applied Mathematics. McGraw-Hill, New York, 1991.

Department of Mathematics, University of Groningen, P.O. Box 800, 9700 AV Groningen, The Netherlands.

E-mail address: R.F.Curtain@math.rug.nl

Department of Mathematics, Royal Institute of Technology, Stockholm 100 44, SWEDEN.

E-mail address: sasane@math.kth.se 